\title{
Inflammasomes and IL-1 biology in the pathogenesis of allograft dysfunction
}

\author{
S. Samuel Weigt, Vyacheslav Palchevskiy, and John A. Belperio \\ Department of Medicine, David Geffen School of Medicine, UCLA, Los Angeles, California, USA.
}

\begin{abstract}
Inflammasomes are high-molecular-weight cytosolic complexes that mediate the activation of caspases. There are many inflammasomes, and each is influenced by a unique pattern-recognition receptor response. Two signals are typically involved in the inflammasome pathways. Signal one involves recognition of pathogen-associated molecular patterns (PAMPs), such as LPS or other colonizing/invading microbes, that interact with TLRs, which induce the downstream production of pro-IL-1及. This is followed by signal two, which involves recognition of PAMPs or damage-associated molecular patterns (DAMPs), such as uric acid or ATP, via NLRP3, which leads to caspase-1-dependent cleavage of pro-IL-1 $\beta$ to active IL-1 $\beta$ and pyroptosis. Ultimately, these two signals cause the release of multiple proinflammatory cytokines. Both PAMPs and DAMPs can be liberated by early insults to the allograft, including ischemia/reperfusion injury, infections, and rejection. The consequence of inflammasome activation and IL-1 expression is the upregulation of adhesion molecules and chemokines, which leads to allograft neutrophil sequestration, mononuclear phagocyte recruitment, and T cell activation, all of which are key steps in the continuum from allograft insult to chronic allograft dysfunction.
\end{abstract}

\section{Introduction}

Multiple studies have suggested that IL-1 has a key role in allograft rejection. The generation of mature, highly bioactive IL- $1 \beta$ requires proteolytic processing of its precursors by the enzyme caspase-1, also known as IL-1 $1 \beta$-converting enzyme (ICE) $(1,2)$. Caspase- 1 is activated through formation of the inflammasome complex. The term inflammasome was coined in the early 2000s to describe a high-molecular-weight complex present in the cytosol of cells that mediated the activation of caspases (3). We now know that there are many inflammasomes and that each one is influenced by a unique pattern-recognition receptor response to a pathogenassociated molecular pattern (PAMP) or a damage-associated molecular pattern (DAMP).

The canonical inflammasome pathway is responsible for activating caspase- 1 in humans and rodents. For example, engagement of NLR, NACHT, LRR, and PYD domains-containing protein 3 (NALP3; also known as cryopyrin), which is encoded by NLRP3 in humans, induces oligomerization and the recruitment of an adaptor protein known as apoptosis-associated speck-like protein containing a caspase recruitment domain (ASC). ASC has two death-fold domains, PYD and CARD, and acts to bridge the sensor molecule, NLRP3, to the effector molecule, pro-caspase-1 (Figure 1). A proximity-induced process results in the formation of the machinery called the NLRP3 inflammasome, which cleaves pro-caspase-1 to generate caspase-1. Caspase- 1 drives the processing of pro-IL-1 $\beta$ to IL-1 $\beta$ as well as that of pro-IL-18 to IL-18. Caspase-1 also induces pyroptosis, a proinflammatory programmed cell death via a process that includes cell swelling, membrane rupture, and release of intracellular inflammatory mediators (refs. 4,5 , and Figure 1).

Conflict of interest: The authors have declared that no conflict of interest exists. Reference information: J Clin Invest. 2017;127(6):2022-2029.

https://doi.org/10.1172/JCI93537.
Multiple receptor proteins are known to induce assembly of canonical inflammasomes. NLRP3 acts as a sensor for many PAMPs and DAMPs. Other examples include NLRP1, which senses anthrax lethal toxin; NLRC4, which senses flagella; and absent in melanoma 2 (AIM2) and pyrin, which sense double-stranded DNA $(4,5)$. Akin to caspase-1 activation by the canonical inflammasome machinery, there is a noncanonical inflammasome, in which cytosolic LPS is detected directly by inflammatory caspases, including caspase- 11 in rodents and caspase- 4 and caspase- 5 in humans. More specifically, LPS binds via its lipid A to the CARD in the caspase. This binding stimulates oligomerization- and proximity-induced activation of caspase-11 in rodents or caspase-4 and caspase- 5 in humans. Activated caspase-11, caspase-4, or caspase-5 leads to pyroptosis as well as the activation of the canonical NLRP3 inflammasome (refs. 6-10 and Figure 1).

\section{Inflammasomes and IL-1 transplant ischemia/ reperfusion injury}

Solid organ ischemia/reperfusion injury (IRI) is a multifactorial process that leads to early allograft dysfunction. In addition to IRI, donor factors that may contribute to early graft dysfunction include brain death and the accompanying neuroendocrine derangements, cardiogenic shock that requires vasopressor support, and mechanical ventilation. The physical trauma associated with organ manipulation during procurement, disruption of lymphatics, and microbial infection may further compound donor factors and organ ischemia. Overall, these different forms of trauma lead to cellular damage and the release of autocrine and paracrine mediators that both initiate and perpetuate inflammatory responses that directly lead to early allograft dysfunction. Many investigations implicate inflammasome pathways during IRI and early allograft dysfunction (11-13). Early studies of inflammasomes in transplantation used the fully mismatched $\mathrm{BALB} / \mathrm{c}$ to $\mathrm{C} 3 \mathrm{H} / \mathrm{He}$ 


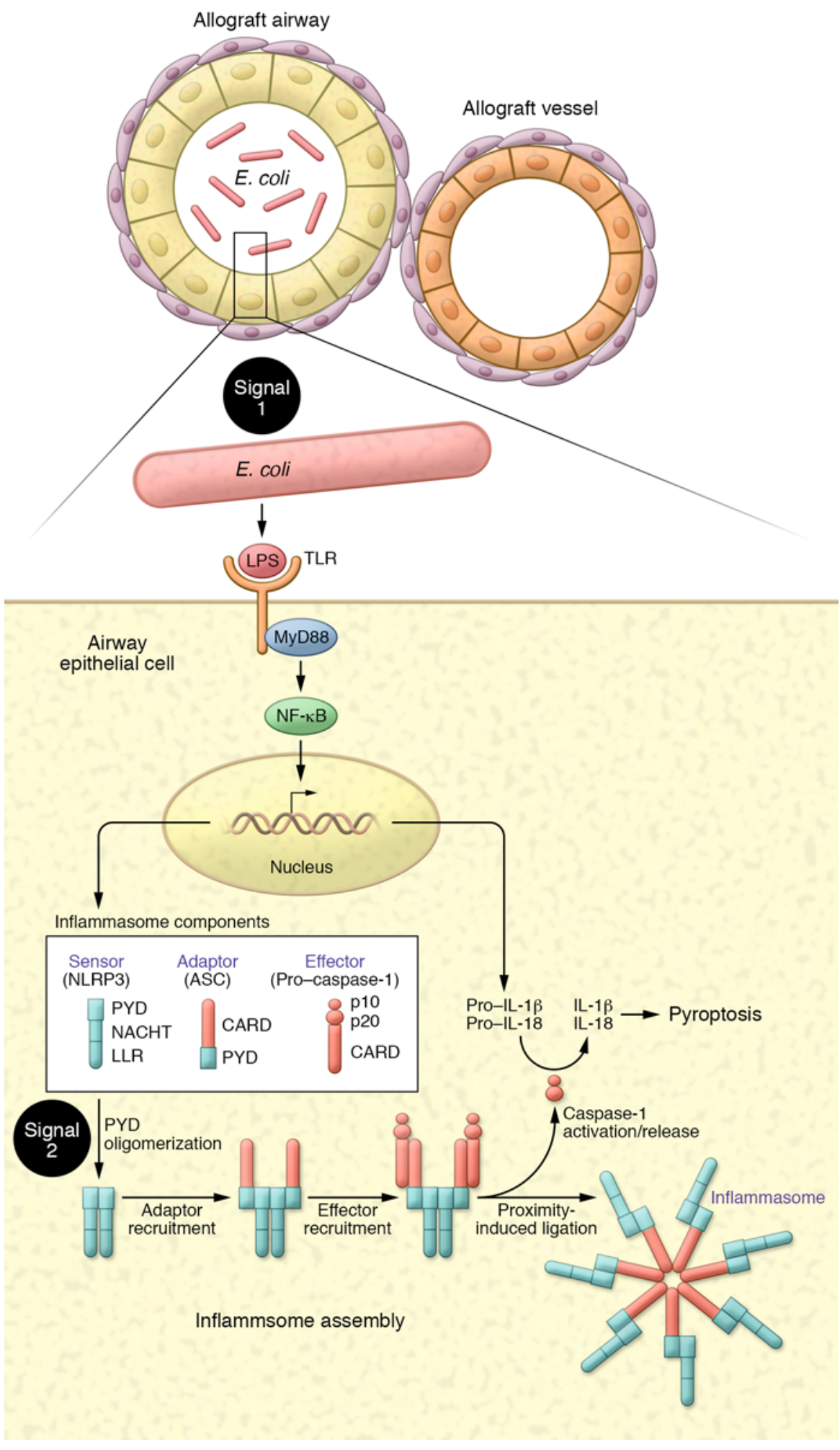

Figure 1. Allograft airway with an adjacent vessel. The allograft airway is prone to colonization and infection with $E$. coli due to transplant immunosuppression and gastroesophageal reflux. The $E$. coli stimulate TLRs via LPS, leading to MyD88 and NF- $\kappa B$ signaling that generates pro-IL-1 $\beta$. Allograft injury from invading E. coli can lead to increased levels of uric acid (UA) that are detected by the cytosolic sensor NLRP3, which contains a pyrin domain (PYD), NACHT, and LLR. This monomeric sensor becomes activated, resulting in oligomerization via PYD homotypic interactions. Next, the ASC adaptor protein, which contains two death-fold domains that include the PYD and the caspase recruitment domain (CARD), is recruited. The ASC adaptor CARD domain allows for binding to the effector caspase via induced proximity, which creates a seven-NLRP3/ASC adaptor protein called the NLRP3 inflammasome. The NLRP3 inflammasome mediates proteolytic cleavage of procaspase- 1 to caspase-1, resulting in pyroptosis, as well as the processing of pro-IL-1 $\beta$ to IL-1 $\beta$. It should be noted that cytoplasmic LPS can be sensed by rodent caspase-11 or human caspase-4 or caspase-5, leading to pyroptosis, and can activate NLRP3. This complex is termed the noncanonical inflammasome.

of inflammasomes, ASC-deficient $\left(\mathrm{Asc}^{-/-}\right)$ and WT mice were compared in a nontransplant warm liver IRI murine model (16). $\mathrm{Asc}^{-/}$mice had a marked reduction in the IL-1-inducible chemokines CXCL1 and CCL2, which led to a reduction in neutrophil sequestration and infiltrating mononuclear phagocytes. Additionally, the livers from $A s c^{-/}$animals had a reduced expression of high-mobility group box 1 (HMGB1), TLR4, NF-кB, and cleaved caspase-1. HMGB1 is an evolutionarily conserved and ubiquitously expressed DNA-binding protein in the nucleus that stabilizes nucleosome formation and can facilitate gene transcription, repair, and recombination (17). In addition to its nuclear role, extracellular HMGB1 is considered an endogenous DAMP that can activate inflammatory pathways. HMGB1 is released by ischemia-stressed cells $(18,19)$ and, when bound to LPS, can engage TLRs and the receptor for advanced glycation end products (RAGE) (20-22). Asc ${ }^{-/}$mice

heterotopic cardiac allograft model. Importantly, ASC expression occurred in the IRI phase for both isografts and allografts (14), which led to further evaluation of the inflammasome pathways during early allograft dysfunction.

Liver IRI involves tremendous leukocyte and Kupffer cell activation, causing a cytokine storm that results in hepatocyte and sinusoidal endothelial cell death (15). To establish the role had a reduction in alanine aminotransferase and liver ischemia/ reperfusion damage. Interestingly, intravenous infusion of superphysiologic levels of HMGB1 induced IRI in $\mathrm{Asc}^{-/}$mice, suggesting that IRI causes release of physiologic HMGB1 that can bind LPS and interact with TLRs. Additionally, IRI also involves the release of other PAMPs and DAMPs that can be sensed by cytoplasmic inflammasome sensors and, via ASC, activates caspase-1- 
mediated IL-1 $\beta$ release. Moreover, these investigators (16) found that ASC permits the perpetuation of a proinflammatory environment via a TLR- and ACS-dependent pathway that activates $\mathrm{NF}-\kappa \mathrm{B}$, thereby producing stores of pro-IL- $1 \beta$ that is cleaved by caspase- 1 to biologically active IL-1 $\beta$. Importantly, this study demonstrated that anti-IL-1 $\beta$ therapy markedly decreased IRI to levels similar to those seen in the $\mathrm{Asc}^{-/-}$mice (16). Collectively, these data imply that inhibition of either ASC or IL-1 $\beta$ can decrease warm liver IRI in animal models, which warrants further study in human solid organ transplantation.

While many studies have demonstrated that immune cells and/or mucosal surfaces play a critical role in inflammasome generation, other studies have shown that inflammasomes in mesenchymal cells can also be involved in cytokine production. For example, during in vivo cardiac ischemia/reperfusion in mice, there is an increase in the expression of NLRP3 and IL- $1 \beta$ in cardiac fibroblasts (23). Inflammasome pathways typically require two signals for full activation. In vitro, TLR activation by LPS acts as

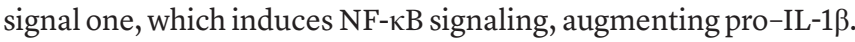
This is followed by exposure to ATP, which can be liberated after tissue IRI and acts as signal two, as it is sensed by NLRP3 and, via ASC, leads to caspase-1-dependent cleavage of pro-IL-1 $\beta$ to active IL-1 $\beta$. Notably, when $N \mathrm{rp}^{-/-}$hearts underwent IRI, there was a profound improvement of cardiac function and a reduction in hypoxic damage as compared with hearts from WT mice. These observations suggest that the sensing of DAMPs and PAMPs by donor mesenchymal cells activates inflammasomes, followed by amplified expression of IL-1 $\beta$, which can then interact with the recipient's immune system, placing the transplant patient at risk for IRI, rejection, and chronic allograft dysfunction.

The most studied inflammasome sensor is NLRP3. NLRP3 is known to be expressed by human renal epithelial cells and leukocytes (24). Human studies involving genotyping of SNPs can be used, to a degree, to determine the effects of genetic variation in the pathobiology of specific diseases and syndromes. To this end, a retrospective cohort study of renal transplant recipients was evaluated for NLRP3 SNPs in donor and recipient DNA to characterize the association with the development of early renal allograft dysfunction. This study specifically evaluated two gain-of-function (GOF) NLRP3 SNPs (R260W and Q705K) and two loss-of-function (LOF) NLRP3 SNPs (rs4353135 and rs6672995) (25). Of note, the GOF SNPs R260W and Q705K are known to enhance IL-1 $\beta$ production and have been linked to Muckle-Wells syndrome, a familial cold autoinflammatory syndrome and chronic infantile neurologic cutaneous and articular syndrome (26-29). The NLRP3 SNP Q705K has also been linked with Crohn's disease, celiac disease, and late-onset Alzheimer's disease (30-32). In a cohort of 1,271 renal transplant recipients, the genotypic distribution of NLRP3 SNPs in donors and recipients was comparable to the general population distributions, and none had a significant deviation from Hardy-Weinberg equilibrium (33). Within the cohort, there were 415 (33\%) cases of delayed graft function (DGF), of which only 60 (14\%) resulted in a primary nonfunctioning graft (PNF). Surprisingly, none of the NLRP3 SNPs in either donor or recipient was associated with the development of DGF or PNF, which may or may not be due to this retrospective study being underpowered.
Lung allograft IRI is associated with primary graft dysfunction (PGD) that is characterized by hypoxemia and radiographic infiltrates within 72 hours of transplantation (34). Approximately $10 \%-30 \%$ of all lung transplant recipients develop clinically significant PGD (35), which is associated with increased short-term and long-term morbidity and mortality $(36,37)$. In the prospective multicenter Clinical Trials in Organ Transplantation-03 (СTOT03) study that included 106 lung transplant recipients, investigators evaluated inflammatory pathways involved in lung IRI (38). Twenty-three (22\%) patients developed grade 3 PGD within 72 hours (i.e., cases), and they were matched one-to-one with twentythree patients that did not develop PGD (i.e., controls). There were no statistically significant differences in donor- or recipient-related characteristics. Transplant type and intraoperative interventions (e.g., cardiopulmonary bypass use or time) were also similar. The study compared differential gene expression in bronchoalveolar lavage cell pellets between PGD cases and controls. Kyoto Encyclopedia of Genes and Genomes pathway analyses indicated that PGD was associated with upregulation of NLR inflammasomes. Additionally, BioCarta pathway analysis revealed that the IL-1 receptor (IL-1R) pathway was markedly upregulated. Furthermore, the highest ranked individual transcripts included IL1B, NLRP3, and IL1A. Importantly, rodent models indicate that warm lung IRI is mediated, in part, by an IL-1R axis (39). Collectively, these studies support the rationale for a human clinical trial that pharmacologically inhibits the inflammasome, which should inhibit the IL-1 pathway in the perioperative lung transplant setting.

In a follow-up study, the CTOT-03 cohort was also utilized to study recipient biologic responses during PGD (40). The investigators evaluated longitudinal peripheral blood leukocyte gene expression. Eighteen genes were differentially expressed in PGD cases, with significant representation of genes involved in both innate and adaptive immunity. Most of the differential expression was a result of early differences in gene expression, with later time points showing no significant differences. Importantly, expression of NLRP3 and NLRC4 was prominently upregulated in PGD cases within the first seven days after transplant as well as over the first year. These results add further support to a role for inflammasomes in PGD. Collectively, the above studies suggest that both donor allograft and the recipient's immune responses to the allograft are influenced by the inflammasome(s) and the downstream IL-1 pathway. Pharmacological inhibition of these pathways after solid organ transplant could potentially lead to better patient outcomes.

\section{Inflammasomes and IL-1 biology in the pathogenesis of transplant rejection}

Inflammasome pathways have high potential to be key players in acute and chronic allograft rejection (11-13). One of the early studies of inflammasomes in transplantation found, in a fully mismatched model of cardiac rejection, that there was a noticeable increase in ASC expression from heart allografts with rejection as compared with isograft controls, and this expression pattern paralleled IL-1 $\beta$ expression (14). These investigations suggested that there is a possibility that inflammasomes may play key roles in the pathobiology of rejection. Similar to solid organ transplantation, the role of inflammasomes during allogeneic hematopoietic stem cell transplantation (allo-HSCT) 
is also being intensely studied. During allo-HSCT, donor T cells present in the allograft target host tissues and cause graftversus-host disease (GvHD) (41). Conditioning regimens include cytotoxic drugs and $\gamma$-irradiation to facilitate marrow engraftment; however, these regimens can also lead to tissue damage. In conjunction with the profound reduction in hematopoietic cells, injury to the GI tract allows for bacterial translocation, which initiates and perpetuates a cytokine storm that may contribute to the activation of recipient antigen-presenting cells and donor $\mathrm{T}$ cells, resulting in GvHD (42). Using a murine model of GvHD (BALB/c mice conditioned with total body irradiation, with or without cytotoxic chemotherapy, followed by C57BL/6 bone marrow $[\mathrm{BM}]$ transplant), administration of a recombinant IL-1R antagonist (IL-1RA) prior to conditioning attenuated GvHD and improved survival, as compared with control therapy or delayed therapy started five days after conditioning (43). The sources of IL-1 $\beta$ were DCs, myeloid cells, fibroblasts, and endothelial cells. The depletion of intestinal microbial flora decreased IL-1 $\beta$ expression from both the intestine and skin, supporting the concept that gut microflora load may influence GvHD. Additionally, the conditioning regimen led to increased levels of the DAMPs uric acid (UA) and ATP. Notably, preconditioning therapy with uricase, which decreases UA levels, or the inhibition of ATP binding to its cellular receptor P2X7 reduced IL-1 $\beta$ expression and attenuated $\operatorname{GvHD}(43,44)$. IL-1R was found to be expressed on allogeneic BM DCs and T cells, and the use of IL-1R-deficient T cells or DCs delayed GvHD (43).

Given the importance of NLRP3 as an inflammasome sensor for multiple DAMPs and PAMPS $(4,5,45)$, investigators used $\mathrm{Asc}^{-1-}$ and $\mathrm{Nlrp3}^{-/-}$mice in a model of GvHD. They found that $\mathrm{Asc}^{-/-}$and $\mathrm{Nlrp}^{-/-}$recipient mice exhibited delayed onset of GvHD, similarly to mice treated with IL-1RA (43). Conversely, transplantation with $\mathrm{Asc}^{-/}$or $\mathrm{Nlrp}^{-/-}$donor BM did not delay the onset of GvHD (43). This study highlights the two signals that activate the NLRP3 inflammasome in the pathogenesis of GvHD (refs. 43, 44, and Figure 1). Signal one is a priming signal involving the recognition of PAMPs (e.g., gastrointestinal microflora) interacting with TLRs to cause upregulation of pro-IL-1 $\beta$. This is followed by signal two, which involves recognition of DAMPS (e.g., UA and ATP) by NLRP3, which leads to caspase-1-dependent cleavage of pro-IL-1 $\beta$ to active IL-1 $\beta$. Importantly, LPS-stimulated human peripheral blood leukocytes from patients with GvHD intestinal lesions exhibited increased levels of cleaved caspase- 1 and IL-1 $\beta$, indicating that inflammasome activation may also be germane to human GvHD.

Myeloid-derived suppressor cells (MDSCs) are broadly defined as myeloid lineage cells with immune-suppressive capacity $(46,47)$. With regard to allo-HSCT, donor MDSCs can be enriched from fresh donor BM treated with granulocyte macrophage-CSF and granulocyte-CSF, followed by IL-4 and IL-13 (referred to as MDSC-IL-13) $(46,47)$. When lethally irradiated mice underwent allo-HSCT with CD25-depleted T cells plus MDSC-IL-13 therapy at day zero, there was a modest delay in the development of GVHD, but this delay was not sustained $(46,48)$. Congenic MDSC-IL-13 cells did not exhibit a reduction in frequency or viability (46). The MDSC-IL-13 subset that was recovered during the development of GvHD showed a potential increase in the inflammasome pathway, as suggested by an upregulation of caspase-1 p10 and IL-1 3 . Furthermore, inflammasome activation via NLRP3 (through treatment with LPS and ATP) or AIM2 (through treatment with LPS and oligo-deoxythymidine [poly(dT)]) inhibited the ability of MDSC-IL-13 to delay GvHD. Conversely, when $\mathrm{Asc}^{-/-}$ MDSC-IL-13 were studied ex vivo, neither LPS challenge (signal one) nor ATP or poly(dT) challenge (signal two) induced IL-1 $\beta$, and the cells maintained their ability to regulate $\mathrm{T}$ cell proliferation. Moreover, inhibiting the inflammasome within MDSC-IL-13 cells repressed their plasticity in vivo and sustained their regulatory functional capacity. Collectively, these findings suggest an important role for inflammasomes in the continuum from innate (conditioning and/or IRI) to adaptive (acute to chronic rejection) immunity and in limiting the potential of MDSC-IL-13 therapy in an allo-environment.

Inflammasome sensing leads to the assembly and generation of the large, multiprotein ASC complex, which can be labeled and visualized via light microscopy $(49,50)$. A small exploratory study was performed on endomyocardial biopsies from heart transplant recipients $(n=8)$ with rejection (51). All patients in this cohort went on to die within two years of biopsy (2-663 days), and the causes of death included sepsis $(n=1)$, heart failure $(n=3)$, and sudden death $(n=4)$. ASC detection by high-power field light microscopy demonstrated that ASC specks were localized to leukocytes and cardiomyocytes in the biopsies from 7 of the 8 heart transplant patients. They found a temporal relationship between increased ACS speck-positive cells and rejection grade. This study was limited by small numbers, possible sampling errors, an all-male patient cohort, and deaths within two years of the biopsies; thus, an appropriate validation study is needed. Nonetheless, these exploratory data are compelling in linking inflammasomes with the development of acute rejection in human cardiac transplant recipients.

The role of the NLRP3 inflammasome in acute rejection was further explored in a retrospective study involving 1,271 renal transplant patients. The overall cumulative incidence of biopsyproven acute rejection (BPAR) in this cohort was 34\% (433 of 1,271) (33). Ninety-one percent (393 of 433) of the BPAR occurred in the first year of transplant and 9\% (40 of 433) occurred after the first year (termed late BPAR). The donor NLRP3 SNP rs35829419, which has a GOF phenotype, was associated with an increased risk for BPAR (hazard ratio 1.48, 95\% CI 1.12-1.96, $P=0.006$ ). The recipient NLRP3 SNP rs6672995, which has a LOF phenotype, was significantly associated with a decreased risk for BPAR (hazard ratio $0.72,95 \% \mathrm{CI} 0.58-0.91, P=0.005)$. These results suggest that a pharmacologic strategy to reduce NLRP3 function may be a way to profoundly reduce the incidence of acute rejection, which may translate into decreased chronic allograft dysfunction.

IL- $1 \alpha$ is considered to be a DAMP (52) that binds the type 1 IL-1R and recruits the IL-1R accessory protein, forming a complex that interacts with the adaptor protein MyD88. Activation triggers a phosphorylation-mediated signaling cascade that leads to NF- $\mathrm{B}$ activation and upregulation of multiple cytokine genes (2). IL-1 $\alpha$ resides in the cytosol as a $33-\mathrm{kDa}$ precursor, and, when released from injured cells, it acts as a danger signal or alarmin. However, some but not all studies describe precursor IL-1 $\alpha$ as less biologically active than its calpain-cleaved mature form $(53,54)$. Under noninflammatory conditions, human endothelial cells undergoing injury (i.e., simple freeze/thaw) have very low levels of IL-1 $\alpha$ pro- 


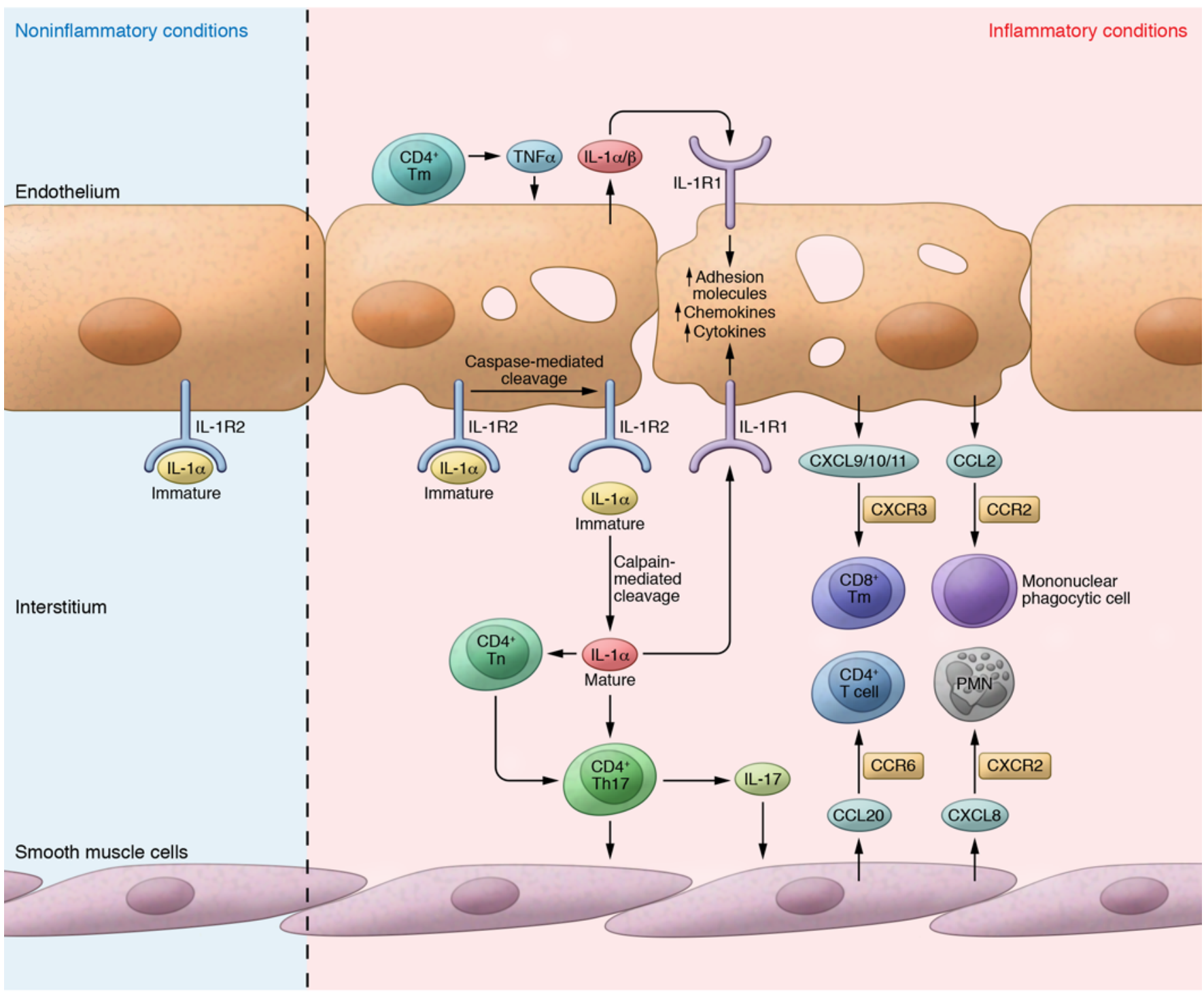

Figure 2. IL-1 and allograft endothelial cells. During normal endothelial cell turnover, the precursor IL-1 $\alpha$ stays bound to IL-1R2 and, when released, is less biologically active than mature IL-1 $\alpha$; thus, there is minimal inflammation that perturbs adjacent endothelial cells. However, when CD4+ memory T cells $\left(C D 4^{+} \mathrm{Tm}\right)$ interact with the allograft endothelium, there is an induction of CD4+ ${ }^{+} \mathrm{Tm}$-secreted TNF- $\alpha$. In turn, TNF- $\alpha$ activates the endothelial cells, allowing caspase to cleave IL-1R2 away from precursor IL-1 $\alpha$, which is then cleaved by calpain to the mature, biologically active IL-1 $\alpha$. IL-1 $\alpha$ skews naive CD4+ $T$ cells ( $C D 4^{+} \mathrm{Tn}$ ) toward a Th17 phenotype. The Th17 cells secrete IL-17, which interacts with smooth muscle cells, augmenting their expression of chemokines that recruit injurious leukocytes. IL-1 also interacts with adjacent endothelial cells, upregulating adhesion molecules, cytokines, and chemokines that allow for the recruitment of more injurious alloreactive cells as well as other mononuclear cells.

tein, and minimal inflammation and injury are induced in adjacent cells. In contrast, endothelial cells subjected to freeze/thaw injury in a proinflammatory environment (e.g., stimulated with TNF- $\alpha$ and IL-1 $1 \beta$ ) do not have altered IL-1 $\alpha$ protein levels, yet IL-1 $\alpha$ biological activity dramatically escalates. Immunofluorescence and proximity ligation assays have demonstrated that the immature form of IL-1 $\alpha$ associates with IL-1R2 (55); however, TNF- $\alpha$ and IL-1 $1 \beta$ induce caspase proteolytic activity, cleaving IL-1R2 away from the immature form of IL-1 $\alpha$. Immature IL- $1 \alpha$ is then processed by calpain to the highly biologically active IL-1 $\alpha$ mature form (ref. 55 and Figure 2).

With regard to transplantation, in vitro studies have demonstrated that human alloreactive $\mathrm{CD} 4^{+} \mathrm{T}$ memory (Tm) cells express TNF- $\alpha$, activating endothelial cells to express biologically active IL- $1 \alpha$, which in turn polarizes $\mathrm{CD} 4^{+} \mathrm{Tm}$ cells toward the production of IL-17 (56-60). Corroborating studies were performed using a human/mouse chimeric model of intima arteritis, in which a short segment of human coronary artery was implanted into SCID/beige mice at the infrarenal aortic interposition (57). The adoptive transfer of allogeneic human peripheral blood mononuclear cells markedly increased IL-1 $\alpha$ expression from the endothelium of the grafts. Inhibiting IL-1 $\alpha$ partially reduced vessel intimal obliteration, lowered the number of infiltrating $\mathrm{T}$ cells, and decreased graft expression of IL-17. Ex vivo experiments suggested that IL-1 $\alpha$ blockade suppressed IL-17-expressing $\mathrm{CD} 4^{+} \mathrm{T}$ cells, thereby limiting IL-17 interaction with smooth muscle cells and 
downregulating their production of IL-6, CXCL8, and CCL20, while attenuating alloreactivity. This study confirmed an important function for IL-1 $\alpha$ via downstream inflammatory pathways in the pathogenesis of allograft dysfunction (Figure 2).

Interestingly, $\mathrm{Illr}^{-1}$ recipient mice did not exhibit altered allograft dysfunction in a murine cardiac heterotopic transplant model (61), but there was a modest improvement of allograft survival using donor grafts from $\mathrm{Illr}^{-/}$mice. During the IRI phase, there was a reduction in CXCL1 and CXCL2, limiting neutrophil sequestration, which is consistent with other recent studies demonstrating the importance of neutrophils and neutrophil extracellular traps during IRI (62-64). The Il1r-- allografts also limited IFN- $\gamma$ expression, consistent with a reduction in infiltrating $\mathrm{CD}^{+} \mathrm{Tm}$ cells. Conversely, there was an increase in infiltrating mononuclear phagocytes with a regulatory expression profile as compared with classically activated mononuclear phagocytes, while there was no change in infiltrating CD $4^{+} \mathrm{Tm}$ cells. Il1 $r^{-/}$donor allografts combined with $\mathrm{CD}^{+} \mathrm{T}$ cell depletion had no effect on the Illr $^{-/}$allograft long-term survival, but CD $4^{+}$ $\mathrm{T}$ cell depletion at day four further improved allograft survival, demonstrating that the $\mathrm{CD} 4^{+} \mathrm{T}$ cells generated during alloinjury from $\mathrm{Illr}^{-/}$donor grafts were injurious to the graft. Chimeric mice were used to determine the influence of hematopoietic and nonhematopoietic Il1r-/ cells on allograft dysfunction. Donor chimeric cardiac grafts (nonhematopoietic $\mathrm{Illr}^{-/}$parenchymal cells with hematopoietic WT BM cells) were transplanted into WT recipients. The recipients exhibited reductions in mononuclear phagocytes, neutrophils, and donor-responsive $\mathrm{CD} 4^{+}$and $\mathrm{CD} 8^{+} \mathrm{T}$ cells. This finding indicates that signals via IL-1R from the donor allograft parenchymal cells can create an allograft environment involving specific cytokines and chemokines that recruit neutrophils and distinct mononuclear phagocytes, which then expand and influence donor-responsive $\mathrm{CD}^{+} \mathrm{T}$ cells to a far greater extent than $\mathrm{CD}^{+} \mathrm{T}$ cells. This study also demonstrates that controlling both $\mathrm{CD}^{+}$and $\mathrm{CD}^{+}$alloresponsive $\mathrm{T}$ cells will be critical for long-term allograft survival.

\section{Conclusion}

Collectively, the studies highlighted in this Review suggest that inflammasomes play a key role in regulating IL-1 biology in the allograft. This process occurs very early in the inflammatory cas- cade that is inherent to multiple forms of allograft injury, rejection, and, ultimately, dysfunction. Both IL-1 $\alpha$ and IL- $1 \beta$ have been shown to be associated with the full spectrum of IRI, from acute rejection to chronic allograft dysfunction $(43,57,58,61,65-68)$. Mechanistically, research suggests that the role of IL-1 in the process of allograft dysfunction is in part initiated by Tm cells interacting with parenchymal cells, causing the release of TNF- $\alpha$ from CD4 ${ }^{+}$Tm cells (56-60). TNF- $\alpha$ induces allograft parenchymal cells to release active IL- $1 \alpha$ via caspases and calpain. IL- $1 \alpha$ then skews T cells toward a Th17 phenotype that releases IL-1 $\beta$ and IL-17. The parenchymal inflammasome is at least partially responsible for upregulation of IL-1 $\beta$ production via signal one and signal two. Active IL-1 $\beta$ interacts with DCs and T cells expressing IL-1R, causing a feed-forward mechanism that results in the production of more TNF- $\alpha$, IL- $1 \alpha$, and IL- $1 \beta$ as well as the upregulation of adhesion molecules, cytokines, and chemokines. Importantly, some of the IL-1-induced chemokine pathways have causal and nonredundant roles in promoting allograft injury, rejection, and dysfunction. More specifically, the CCR2 signaling axis was found to be important for the recruitment of a phenotypically distinct set of profibrotic mononuclear phagocytes (69). CXCR6/CCL20 and CXCR3/ ligands influence Th17 and Th1 lymphocyte responses, respectively (70-73). CXCR2 signaling was found to be important in recruiting neutrophils during IRI $(63)$, infections $(74,75)$, acute rejection (62), and, later on, in promoting the vascular remodeling required to support the fibroplasia of the allograft (62). Given the key proximal role of inflammasomes and IL-1 in the allograft injury process, further attention to other potential inducers, such as microbes, ER stress, and cellular metabolism, as well as pharmacologic manipulation of inflammasomes and IL-1 biology in the pathogenesis of allograft injury, rejection, and dysfunction is warranted.

\section{Acknowledgments}

This study was supported by NIH grants R01HL112990 (PI, JAB) and U01AI063594 (Co-PI, JAB, and coinvestigator, SSW) and the UCLA Clinical and Translational Science Institute UL1TR001881.

Address correspondence to: John A. Belperio, UCLA School of Medicine, 900 Veteran Room, 14-154 Warren Hall, Box 957119, Los Angeles, California 90095-7119, USA. Phone: 310.794.1999; Email: jbelperio@mednet.ucla.edu.
1. Dinarello CA. Interleukin 1 and interleukin 18 as mediators of inflammation and the aging process. Am J Clin Nutr. 2006;83(2):447S-455S.

2. Dinarello CA. Immunological and inflammatory functions of the interleukin-1 family. Annu Rev Immunol. 2009;27:519-550.

3. Martinon F, Burns K, Tschopp J. The inflammasome: a molecular platform triggering activation of inflammatory caspases and processing of proIL-beta. Mol Cell. 2002;10(2):417-426.

4. Broz P, Dixit VM. Inflammasomes: mechanism of assembly, regulation and signalling. Nat Rev Immunol. 2016;16(7):407-420.

5. Man SM, Kanneganti TD. Regulation of inflammasome activation. Immunol Rev. 2015;265(1):6-21.

6. Hagar JA, Powell DA, Aachoui Y, Ernst RK, Miao
EA. Cytoplasmic LPS activates caspase-11: implications in TLR4-independent endotoxic shock. Science. 2013;341(6151):1250-1253.

7. Kayagaki N, et al. Noncanonical inflammasome activation by intracellular LPS independent of TLR4. Science. 2013;341(6151):1246-1249.

8. Knodler LA, et al. Noncanonical inflammasome activation of caspase-4/caspase-11 mediates epithelial defenses against enteric bacterial pathogens. Cell Host Microbe. 2014;16(2):249-256.

9. Meunier E, et al. Caspase-11 activation requires lysis of pathogen-containing vacuoles by IFN-induced GTPases. Nature. 2014;509(7500):366-370.

10. Shi J, et al. Inflammatory caspases are innate immune receptors for intracellular LPS. Nature. 2014;514(7521):187-192.

11. Masumoto J, et al. ASC, a novel 22-kDa protein, aggregates during apoptosis of human promyelocytic leukemia HL-60 cells. J Biol Chem. 1999;274(48):33835-33838.

12. Masumoto J, Taniguchi S, Nakayama K, Ayukawa K, Sagara J. Murine ortholog of ASC, a CARDcontaining protein, self-associates and exhibits restricted distribution in developing mouse embryos. Exp Cell Res. 2001;262(2):128-133.

13. Taniguchi S, Sagara J. Regulatory molecules involved in inflammasome formation with special reference to a key mediator protein, ASC. Semin Immunopathol. 2007;29(3):231-238.

14. Seto T, et al. Upregulation of the apoptosis-related inflammasome in cardiac allograft rejection. J Heart Lung Transplant. 2010;29(3):352-359.

15. Zhai Y, Busuttil RW, Kupiec-Weglinski JW. Liver ischemia and reperfusion injury: new 
insights into mechanisms of innate-adaptive immune-mediated tissue inflammation. Am J Transplant. 2011;11(8):1563-1569.

16. Kamo N, et al. ASC/caspase-1/IL-1 $\beta$ signaling triggers inflammatory responses by promoting HMGB1 induction in liver ischemia/reperfusion injury. Hepatology. 2013;58(1):351-362.

17. Javaherian K, Liu JF, Wang JC. Nonhistone proteins HMG1 and HMG2 change the DNA helical structure. Science. 1978;199(4335):1345-1346

18. Scaffidi P, Misteli T, Bianchi ME. Release of chromatin protein HMGB1 by necrotic cells triggers inflammation. Nature. 2002;418(6894):191-195.

19. Tsung A, et al. HMGB1 release induced by liver ischemia involves Toll-like receptor 4 dependent reactive oxygen species production and calcium-mediated signaling. J Exp Med. 2007;204(12):2913-2923.

20. Ivanov S, et al. A novel role for HMGB1 in TLR9-mediated inflammatory responses to CpGDNA. Blood. 2007;110(6):1970-1981.

21. Park JS, et al. Involvement of Toll-like receptors 2 and 4 in cellular activation by high mobility group box 1 protein. J Biol Chem. 2004;279(9):7370-7377.

22. van Zoelen MA, et al. Role of Toll-like receptors 2 and 4, and the receptor for advanced glycation end products in high-mobility group box 1-induced inflammation in vivo. Shock. 2009;31(3):280-284.

23. Sandanger $\varnothing$, et al. The NLRP3 inflammasome is up-regulated in cardiac fibroblasts and mediates myocardial ischaemia-reperfusion injury. Cardiovasc Res. 2013;99(1):164-174.

24. Vilaysane A, et al. The NLRP3 inflammasome promotes renal inflammation and contributes to CKD. J Am Soc Nephrol. 2010;21(10):1732-1744.

25. Villani AC, et al. Common variants in the NLRP3 region contribute to Crohn's disease susceptibility. Nat Genet. 2009;41(1):71-76.

26. Agostini L, Martinon F, Burns K, McDermott MF, Hawkins PN, Tschopp J. NALP3 forms an IL-1 $\beta$-processing inflammasome with increased activity in Muckle-Wells autoinflammatory disorder. Immunity. 2004;20(3):319-325.

27. Hoffman HM, Mueller JL, Broide DH, Wanderer AA, Kolodner RD. Mutation of a new gene encoding a putative pyrin-like protein causes familial cold autoinflammatory syndrome and Muckle-Wells syndrome. Nat Genet. 2001;29(3):301-305.

28. Hull KM, Shoham N, Chae JJ, Aksentijevich I, Kastner DL. The expanding spectrum of systemic autoinflammatory disorders and their rheumatic manifestations. Curr Opin Rheumatol. 2003;15(1):61-69.

29. Verma D, et al. The Q705K polymorphism in NLRP3 is a gain-of-function alteration leading to excessive interleukin-1 $\beta$ and IL-18 production. PLoS One. 2012;7(4):e34977.

30. Pontillo A, Vendramin A, Catamo E, Fabris A, Crovella S. The missense variation $\mathrm{Q} 705 \mathrm{~K}$ in CIAS1/NALP3/NLRP3 gene and an NLRP1 haplotype are associated with celiac disease. Am J Gastroenterol. 2011;106(3):539-544.

31. Roberts RL, Topless RK, Phipps-Green AJ, Gearry RB, Barclay ML, Merriman TR. Evidence of interaction of CARD8 rs2043211 with NALP3 rs35829419 in Crohn's disease. Genes Immun.
2010;11(4):351-356

32. Tan MS, et al. NLRP3 polymorphisms are associated with late-onset Alzheimer's disease in Han Chinese. J Neuroimmunol. 2013;265(1-2):91-95.

33. Dessing MC, Kers J, Damman J, Navis GJ, Florquin S, Leemans JC. Donor and recipient genetic variants in NLRP3 associate with early acute rejection following kidney transplantation. Sci Rep. 2016;6:36315.

34. Christie JD, et al. Report of the ISHLT Working Group on Primary Lung Graft Dysfunction part II: definition. A consensus statement of the International Society for Heart and Lung Transplantation. J Heart Lung Transplant. 2005;24(10):1454-1459.

35. Lee JC, Christie JD, Keshavjee S. Primary graft dysfunction: definition, risk factors, short- and long-term outcomes. Semin Respir Crit Care Med. 2010;31(2):161-171.

36. Daud SA, et al. Impact of immediate primary lung allograft dysfunction on bronchiolitis obliterans syndrome. Am J Respir Crit Care Med. 2007;175(5):507-513.

37. DerHovanessian A, et al. The role of TGF- $\beta$ in the association between primary graft dysfunction and bronchiolitis obliterans syndrome. Am J Transplant. 2016;16(2):640-649.

38. Cantu E, et al. Gene set enrichment analysis identifies key innate immune pathways in primary graft dysfunction after lung transplantation. Am J Transplant. 2013;13(7):1898-1904.

39. Krishnadasan B, Naidu BV, Byrne K, Fraga C, Verrier ED, Mulligan MS. The role of proinflammatory cytokines in lung ischemiareperfusion injury. J Thorac Cardiovasc Surg. 2003;125(2):261-272.

40. Diamond JM, et al. Peripheral blood gene expression changes associated with primary graft dysfunction after lung transplantation [published online ahead of print January 24, 2017]. Am J Transplant. https://doi.org/10.1111/ajt.14209.

41. Ferrara JL, Levine JE, Reddy P, Holler E. Graft-versus-host disease. Lancet. 2009;373(9674):1550-1561.

42. Shlomchik WD. Graft-versus-host disease. Nat Rev Immunol. 2007;7(5):340-352.

43. Jankovic D, et al. The Nlrp3 inflammasome regulates acute graft-versus-host disease. J Exp Med. 2013;210(10):1899-1910.

44. Wilhelm K, et al. Graft-versus-host disease is enhanced by extracellular ATP activating P2X7R. Nat Med. 2010;16(12):1434-1438.

45. Latz E, Xiao TS, Stutz A. Activation and regulation of the inflammasomes. Nat Rev Immunol. 2013;13(6):397-411.

46. Koehn BH, et al. GVHD-associated, inflammasome-mediated loss of function in adoptively transferred myeloid-derived suppressor cells. Blood. 2015;126(13):1621-1628.

47. Koehn BH, Zeiser R, Blazar BR. Inflammasome effects in GvHD. Oncotarget. 2015;6(36):38444-38445.

48. Highfill SL, et al. Bone marrow myeloid-derived suppressor cells (MDSCs) inhibit graft-versus-host disease (GVHD) via an arginase-1-dependent mechanism that is up-regulated by interleukin-13 . Blood. 2010;116(25):5738-5747.

49. Mezzaroma E, et al. The inflammasome pro- motes adverse cardiac remodeling following acute myocardial infarction in the mouse. Proc Natl Acad Sci U S A. 2011;108(49):19725-19730.

50. Stutz A, Horvath GL, Monks BG, Latz E. ASC speck formation as a readout for inflammasome activation. Methods Mol Biol. 2013;1040:91-101.

51. Shah KB, Mauro AG, Flattery M, Toldo S, Abbate A. Formation of the inflammasome during cardiac allograft rejection. Int J Cardiol. 2015;201:328-330.

52. Zheng Y, Gardner SE, Clarke MC. Cell death, damage-associated molecular patterns, and sterile inflammation in cardiovascular disease. Arterioscler Thromb Vasc Biol. 2011;31(12):2781-2786.

53. Afonina IS, et al. Granzyme B-dependent proteolysis acts as a switch to enhance the proinflammatory activity of IL-1 $\alpha$. Mol Cell. 2011;44(2):265-278.

54. Zheng Y, Humphry M, Maguire JJ, Bennett MR, Clarke MC. Intracellular interleukin-1 receptor 2 binding prevents cleavage and activity of interleukin-1 $\alpha$, controlling necrosis-induced sterile inflammation. Immunity. 2013;38(2):285-295.

55. Burzynski LC, Humphry M, Bennett MR, Clarke MC. Interleukin- $1 \alpha$ activity in necrotic endothelial cells is controlled by caspase-1 cleavage of interleukin-1 receptor-2: implications for allograft rejection. J Biol Chem. 2015;290(41):25188-25196

56. Choi J, Enis DR, Koh KP, Shiao SL, Pober JS. T lymphocyte-endothelial cell interactions. Annu Rev Immunol. 2004;22:683-709.

57. Rao DA, et al. Interleukin (IL)-1 promotes allogeneic $\mathrm{T}$ cell intimal infiltration and IL-17 production in a model of human artery rejection. JExp Med. 2008;205(13):3145-3158.

58. Rao DA, Tracey KJ, Pober JS. IL-1alpha and IL-1beta are endogenous mediators linking cell injury to the adaptive alloimmune response. J Immunol. 2007;179(10):6536-6546.

59. Shiao SL, Kirkiles-Smith NC, Shepherd BR, McNiff JM, Carr EJ, Pober JS. Human effector memory $\mathrm{CD}^{+} \mathrm{T}$ cells directly recognize allogeneic endothelial cells in vitro and in vivo. J Iтmunol. 2007;179(7):4397-4404.

60. van Beelen AJ, et al. Stimulation of the intracellular bacterial sensor NOD2 programs dendritic cells to promote interleukin-17 production in human memory T cells. Immunity. 2007;27(4):660-669.

61. Iida S, et al. IL-1 receptor signaling on graft parenchymal cells regulates memory and de novo donor-reactive CD8 T cell responses to cardiac allografts. J Immunol. 2016;196(6):2827-2837.

62. Belperio JA, et al. Role of CXCR2/CXCR2 ligands in vascular remodeling during bronchiolitis obliterans syndrome. JClin Invest. 2005;115(5):1150-1162.

63. Belperio JA, et al. CXCR2/CXCR2 ligand biology during lung transplant ischemia-reperfusion injury. J Immunol. 2005;175(10):6931-6939.

64. Sayah DM, et al. Neutrophil extracellular traps are pathogenic in primary graft dysfunction after lung transplantation. Am J Respir Crit Care Med. 2015;191(4):455-463.

65. Borthwick LA, et al. Pseudomonas aeruginosa induced airway epithelial injury drives fibroblast activation: a mechanism in chronic lung allograft dysfunction. Am J Transplant. 2016;16(6):1751-1765. 
66. Park MJ, et al. IL-1 Receptor blockade alleviates graft-versus-host disease through downregulation of an interleukin-1 $\beta$-dependent glycolytic pathway in Th17 cells. Mediators Inflamm. 2015;2015:631384.

67. Fanslow WC, et al. Regulation of alloreactivity in vivo by a soluble form of the interleukin-1 receptor. Science. 1990;248(4956):739-742.

68. Simeoni E, et al. Gene transfer of a soluble IL-1 type 2 receptor-Ig fusion protein improves cardiac allograft survival in rats. Eur J Cardiothorac Surg. 2007;31(2):222-228.

69. Belperio JA, et al. Critical role for the chemo- kine MCP-1/CCR2 in the pathogenesis of bronchiolitis obliterans syndrome. JClin Invest. 2001;108(4):547-556.

70. Belperio JA, et al. Critical role for CXCR3 chemokine biology in the pathogenesis of bronchiolitis obliterans syndrome. J Immunol. 2002;169(2):1037-1049.

71. Belperio JA, et al. Role of CXCL9/CXCR3 chemokine biology during pathogenesis of acute lung allograft rejection. J Immunol. 2003;171(9):4844-4852.

72. Shino MY, et al. CXCR3 ligands are associated with the continuum of diffuse alveolar damage to chronic lung allograft dysfunction. Am J Respir Crit Care Med. 2013;188(9):1117-1125.

73. Weigt SS, et al. CXCR3 chemokine ligands during respiratory viral infections predict lung allograft dysfunction. Am J Transplant. 2012;12(2):477-484.

74. Gregson AL, et al. Staphylococcus via an interaction with the ELR+ CXC chemokine ENA-78 is associated with BOS. Am J Transplant. 2015;15(3):792-799.

75. Gregson AL, et al. Interaction between Pseudomonas and CXC chemokines increases risk of bronchiolitis obliterans syndrome and death in lung transplantation. Am J Respir Crit Care Med. 2013;187(5):518-526. 